\title{
Editorial
}

\section{Advanced Nanomaterials for Catalysis: Synthesis, Characterization, and Application}

\author{
Xianwen Zhang, ${ }^{1,2}$ Xin Zhang, ${ }^{3}$ Baojuan $\mathrm{Xi},{ }^{4}$ and Maofeng Zhang \\ ${ }^{1}$ Institute of Advanced Energy Technology and Equipment, Hefei University of Technology, Hefei 230009, China \\ ${ }^{2}$ Texas Tech University, Lubbock, TX 79409, USA \\ ${ }^{3}$ Pacific Northwest National Laboratory, Richland, WA 99352, USA \\ ${ }^{4}$ National University of Singapore, Singapore 119077 \\ ${ }^{5}$ Chinese Academy of Sciences, Hefei 230031, China
}

Correspondence should be addressed to Xianwen Zhang; xianwen.zhang@ttu.edu

Received 8 October 2015; Accepted 8 October 2015

Copyright (C) 2015 Xianwen Zhang et al. This is an open access article distributed under the Creative Commons Attribution License, which permits unrestricted use, distribution, and reproduction in any medium, provided the original work is properly cited.

A great attention is being paid to catalysts which act to enhance the rate or yield of the reaction without being consumed by the reaction itself. Catalysis has become a key strategy in solving many of today's challenges. Up to now, almost all industrial processes employ catalyst in multiple forms to increase economic viability, from batteries to refineries. Meanwhile, increased concerns about environmental pollution have led to the development of novel catalysts, which can catalyze desirable chemical reactions. Catalysis is rather prevalent everywhere as a key enabling technology in energy conversion, fuel production, production of chemicals, and environmental mitigation. On the other hand, nanotechnology has become an indispensable tool of material engineering for catalysis related applications. Nanoparticles with controlled morphology and structure have a higher surface area, which provide the best platform to catalyze desirable chemical transformations and lead to the increased catalytic activity. The current barrier for widespread industrial and environmental use is ascribed to the controlled synthesis of nanocrystals with high-energy surfaces and the understanding of synthesis-structure-performance relationships. Multidisciplinary advances from chemistry, physics, and materials science have played a major role in catalyst synthesis, structural and compositional modification, and mechanistic understanding with molecular and atomic-level precision by advanced characterization techniques and modeling.
Last year, we invited investigators to contribute original research as well as review articles that address the field of advanced nanocatalysts. We encourage manuscripts that will concern new progress in controlled synthesis, thorough understanding of the reaction mechanism and cutting-edge characterization techniques. Today, we are very happy to publish this special issue of this journal.

In this issue, original research and review articles are accepted. The research papers focused on nanocatalysts with novel structure produced by innovative synthetic technologies and detailed kinetic mechanism through experiments and promising application.

\section{Acknowledgment}

We would like to thank all authors who submitted their work to this special issue.

Xianwen Zhang

Xin Zhang

Baojuan Xi

Maofeng Zhang 

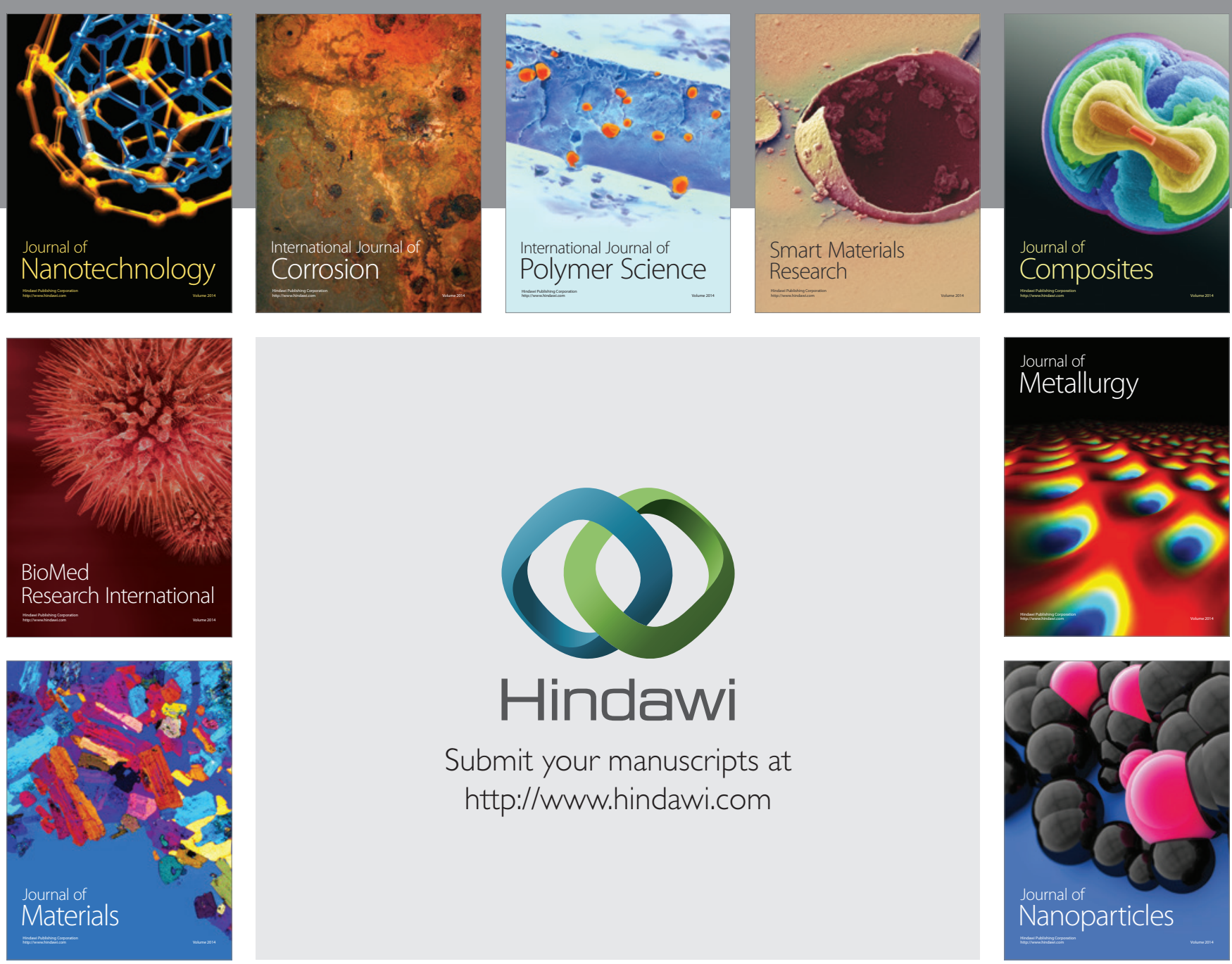

Submit your manuscripts at http://www.hindawi.com
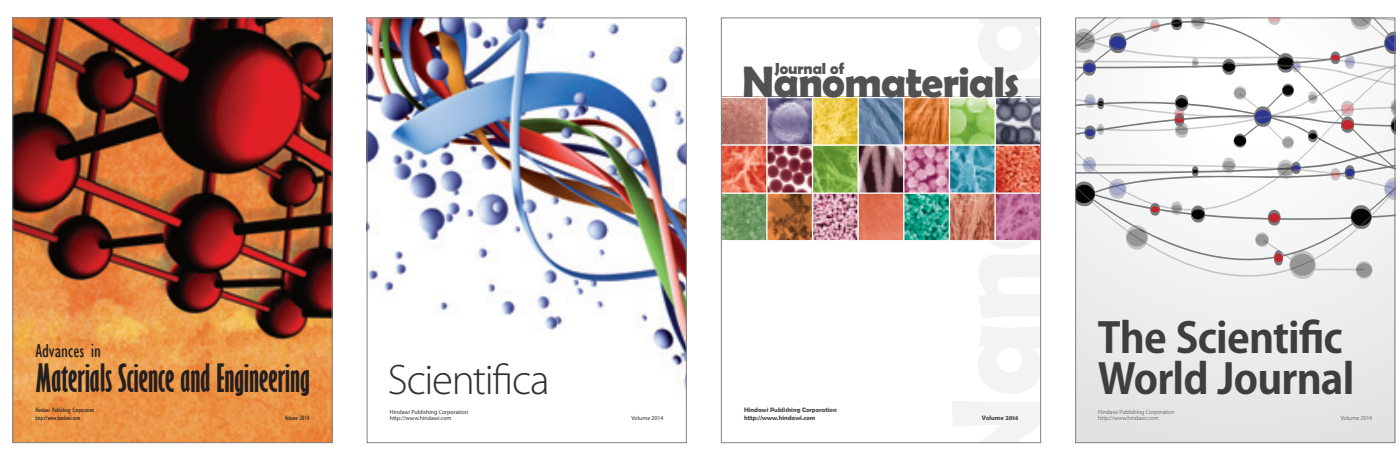

\section{The Scientific World Journal}
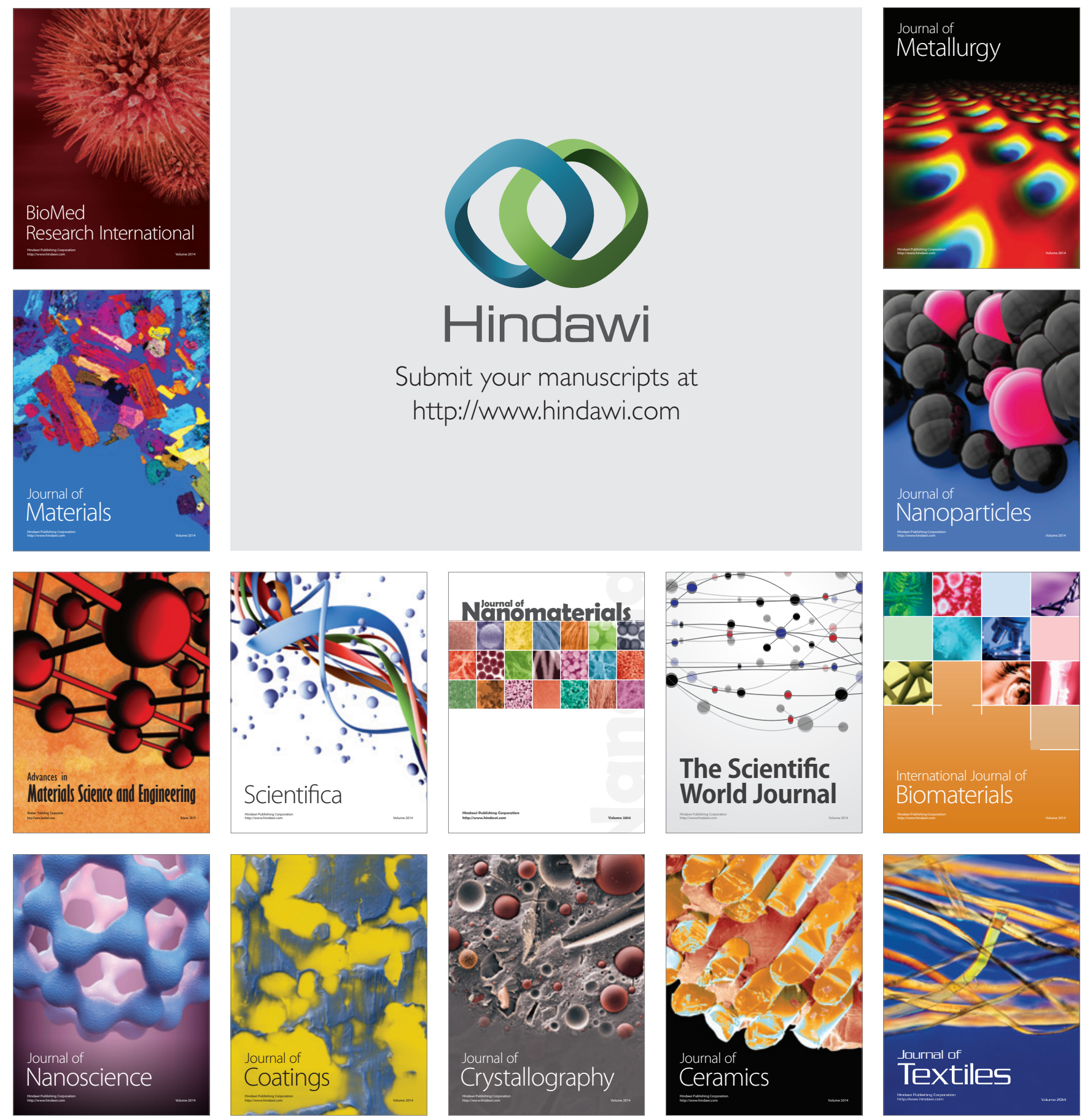